$\stackrel{N / N}{=}$

Global journals Inc.

औf

\title{
Predictors of Depression and Well-Being in Caregivers of Young Children with Developmental Delays in Vietnam
}

By Son Nguyen Duc, Jin Y. Shin, Karleigh Groves, Martha Chaiken \& Amanda Leonard Hofstra University Abstract- The present study examined the impact of social support and other variables on depression experienced by the caregivers of young children with developmental delays in Vietnam. We conducted a survey of 109 caregivers of children with developmental delays who were enrolled in kindergarten programs in Hanoi, Vietnam. The survey included questionnaires on the availability of informal and professional support, perceived social support, and depression. The results suggest that the more the caregivers felt that they received support, the less depression they experienced. However, the amount of informal or professional support was not significantly related to depression. The more maladaptive behaviors their children manifested, the more depressed the caregivers were. The findings suggest that there is a need for services and supports that not only help caregivers to be effective parents but also address feelings of distress that stem from parenting children with disabilities.

Keywords: children with developmental delays, caregivers of children with developmental delays, developmental disabilities, social support, depression, Vietnam.

GJMR-A Classification: NLMC Code: WY 90

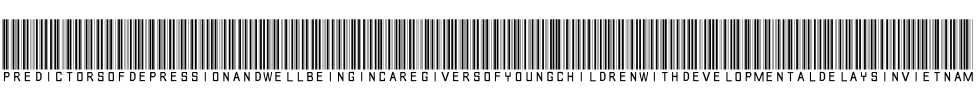

Strictly as per the compliance and regulations of:

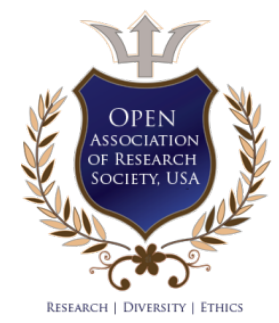

(c) 2021. Son Nguyen Duc, Jin Y. Shin, Karleigh Groves, Martha Chaiken \& Amanda Leonard. This is a research/review paper, distributed under the terms of the Creative Commons Attribution-Noncommercial 3.0 Unported License http://creativecommons.org/licenses/by-nc/3.0/), permitting all non-commercial use, distribution, and reproduction in any medium, provided the original work is properly cited. 


\title{
Predictors of Depression and Well-Being in Caregivers of Young Children with Developmental Delays in Vietnam
}

\author{
Son Nguyen Duc ${ }^{\alpha}$, Jin Y. Shin ${ }^{\sigma}$, Karleigh Groves ${ }^{\circ}$, Martha Chaiken $^{\omega}$ \& Amanda Leonard ${ }^{*}$
}

\begin{abstract}
The present study examined the impact of social support and other variables on depression experienced by the caregivers of young children with developmental delays in Vietnam. We conducted a survey of 109 caregivers of children with developmental delays who were enrolled in kindergarten programs in Hanoi, Vietnam. The survey included questionnaires on the availability of informal and professional support, perceived social support, and depression. The results suggest that the more the caregivers felt that they received support, the less depression they experienced. However, the amount of informal or professional support was not significantly related to depression. The more maladaptive behaviors their children manifested, the more depressed the caregivers were. The findings suggest that there is a need for services and supports that not only help caregivers to be effective parents but also address feelings of distress that stem from parenting children with disabilities.
\end{abstract}

Keywords: children with developmental delays, caregivers of children with developmental delays, developmental disabilities, social support, depression, Vietnam.

\section{INTRODUCTION}

$\mathrm{P}$ arents of children with disabilities experience increased parenting stress due to the caregiving demands the children place on them. Not only do the children fall behind their peers intellectually, but to the extent that their behaviors are maladaptive and inappropriate for their age norms, this will place demands on the caregivers who must learn special ways of raising them. As a result of parenting stress, caregivers of children with disabilities are at an increased risk for depressive symptoms compared to other groups ${ }^{1-5}$.

Author a: Ph.D, Department of Psychology and Pedagogy, Hanoi National University of Education, Hanoi, Vietnam.

e-mail: nguyensonthnue.edu.vn@gmail.com

Corresponding Author o: Ph.D., Department of Psychology, Hofstra University Hempstead, NY, USA.

e-mail:jin.y.shin@hofstra.edu

Author $\rho$ ¥: B.A., Department of Psychology, Hofstra University, Hempstead, NY, USA. e-mails: kgroves1@pride.hofstra.edu,

aleonard2@pride.hofstra.edu

Author w: Ph.D., Department of Psychology, Hofstra University, Hempstead, NY, USA. e-mail: marthaleah.chaiken@hofstra.edu
Olsson and $\mathrm{Hwang}^{3}$ examined whether the increased stress among parents caring for children with disabilities has negative effects on their mental health. Two hundred sixteen participants were recruited using letters mailed to families enrolled in community-based programs for families with disabled children in Sweden. Of the 216 families, 151 children were diagnosed with an intellectual disability without autism, and 65 children were diagnosed primarily with autism. The control group was composed of 214 families with typically developing children of the same geographical area, age, and gender distribution, which the researchers identified using the National Office of Statistics. They found that depressive symptoms were more common among the mothers of children with disabilities than those of children without disabilities. In addition, mothers of children with autism had the highest levels of depression compared to mothers of children with intellectual disabilities without autism, who in turn had higher levels of depression than the control group. Elevated depression scores beyond the cut-off point for clinical depression based on a standardized scale for depression were more common in mothers of children with autism than in mothers of children with intellectual disabilities without autism, who had more elevated scores than the control mothers. Mothers of children with disabilities had overall higher depression scores than fathers of children with disabilities.

Zeedyk and Blacher ${ }^{5}$ considered the different impacts that child disability and child behaviors have on maternal depression over time. The participants included 223 families of children with and without intellectual disabilities drawn from a large multisite study across three different universities in Southern California and Pennsylvania in the U.S. The children were three years old when the families were recruited. They were followed up as the children moved through adolescence. This study found that over the long term, child behavior problems made a greater contribution to maternal depressive symptoms than did child disability status. The study also found that greater child behavior problems, higher financial impact, and lower levels of dispositional optimism were significantly related to higher initial depressive symptoms for mothers. However, only increased child behavior problems had a significant impact on changes to ongoing depressive 
symptoms. As the child moved into adolescence, they found that prior levels of depression for the parent and financial impact were related to greater depressive symptoms.

Support from others can help relieve the psychological and physical strain caregivers experience. The types of support could range from the education provided by teachers, babysitting provided by neighbors, or the willingness of friends to listen to the concerns the caregivers have about their children. It has been widely documented that social support influences the successful adaptation of such families who have children with disabilities and buffers the stress they experience in caring for children with disabilities ${ }^{1,6,7}$.

Zaidman-Zait et al. ${ }^{8}$ studied mothers of children with autism spectrum disorder by compiling data at the time of the child's diagnosis with autism spectrum disorder and then again after two years. The data were drawn from Pathways in ASD, a large Canadian multisite longitudinal study examining the developmental trajectories of children with autism spectrum disorder. When the cohort of 283 mothers was recruited, the children's ages ranged from 2 to 4, and they had been recently diagnosed with ASD. The study found that higher levels of perceived social support by mothers were correlated to lower levels of stress at the time of diagnosis. After two years, it was found that high parenting stress at the time of the child's diagnosis was predictive of increased parenting stress, but higher levels of perceived social support at the time of diagnosis and increased perceived support over time were predictive of decreased parenting stress after two years.

Halstead et al. ${ }^{7}$ identified behavioral and emotional problems of children with developmental disabilities as significant stressors for family members. They also examined whether perceived social support, positive perceptions or coping style could explain some of the variability in the stress the mothers of these children experienced. Participants consisted of 138 mothers whose 4- to 18-year-old children were diagnosed with either autism, Down syndrome or cerebral palsy in England. They found that perceived social support was the only moderating factor, with a statistically significant relationship with life satisfaction, depression, and positive affect for mothers of children with developmental disabilities.

A study conducted by Oh and Lee ${ }^{9}$ examined the relationship between social support and caregiver burden in South Korean mothers of children with developmental disabilities. The participants in this study were recruited from different special education programs, community welfare centers, mental health agencies, and local parents in Seoul, totaling 187 mothers of children ( $\mathrm{M}=10.35$ years) with developmental disabilities. Results indicated that the mothers had strong positive perceptions of emotional support from family and friends; however, they perceived low levels of social support in terms of tangible assistance and immediate help. Furthermore, participants reported perceiving low levels of social support from extended family, friends, social groups, and service organizations. Mothers reported positive contributions and high levels of support from their children's fathers, which was moderately correlated with low levels of caregiver burden. The results showed that social support explained the greatest variance in burden compared to child disability characteristics and mother's socio-demographic characteristics.

Like many other countries in Asia, Vietnam is a collectivistic culture in which filial piety is respected and a greater emphasis is placed on interdependent relationships than on the independence and autonomy of individuals ${ }^{10}$. Often, individuals are expected to sacrifice their needs for the benefit of the group and to meet collectivistic goals. For example, children are expected to do well in school to meet the expectations of their parents, and wives are expected to raise children to meet the expectations of their families. When they fail to do so, they often experience blame and guilt. Negative perceptions and attitudes prevail toward individuals with disabilities, and these are often extended to their mothers and families as not raising their children properly ${ }^{10,11}$.

A few research studies conducted in Vietnam have found that Vietnamese parents of children with intellectual disabilities are stressed 12,13. Vietnamese mothers of young children with developmental delays are more stressed than those of children without delays ${ }^{12}$. Shin et al. ${ }^{11}$ examined parenting stress experienced by mothers and fathers of young children with cognitive delays in Vietnam. The participants consisted of 106 mothers and 93 fathers in Hue city whose children met the criteria of cognitive delays based on the opinions of kindergarten teachers who worked with them and a screening test. They found that mothers were more stressed than fathers. Neither the availability of informal nor professional support was relatedto parenting stress for mothers, but fathers who reported greater availability of informal and professional support experienced less parenting stress. Perceived social support was not related to parenting stress for either mothers or fathers.

In this study, we examined the experience of depression among the caregivers of children with developmental delays in Vietnam as related to social support. As a result of ongoing parenting stress while adequate educational and support systems are not yet fully in place, caregivers might be at risk for depressive symptoms. Depression occurs when the ongoing challenges of caregiving become too overwhelming. With no one to turn to for adequate help, the caregivers begin to wear down and to lose hope and a sense of meaning in life. It was speculated that the ongoing 
parenting stress of coping with their children may wear the caregivers down and make them struggle with the fundamental existential meaning of their lives rather than deriving joy from life and feeling pride in raising their children. It was hypothesized that caregivers who received higher levels of social support would experience a lower level of depression. Social support was assessed in terms of the perception and availability of informal and professional support.

\section{MeTHOD}

\section{a) Participants}

The participants in the study were 109 caregivers of children with developmental delays from Hanoi in Vietnam. Demographic characteristics of the children and caregivers are provided in Table 1. We recruited 116 children with developmental delays and their families from kindergarten programs, which are equivalent to preschool and kindergarten programs in the U.S. These children were identified by their classroom teachers as having developmental delays and being intellectually slower than their peers, based on the teachers' observations. All the children were evaluated with the Scales of Independent BehaviorRevised Early Development Form (SIB-R $)^{14}$, and six children who scored above the $40^{\text {th }}$ percentile were omitted from the study. A total of 109 caregivers whose children's ages ranged from 3 to 8 were included in the data analyses. Forty-two of the caregivers (38.5\%) did not have information on the diagnosis of their children. Among the 67 caregivers (61.5\%) who had such information, 11 (10.1\%) had children who had been diagnosed with ADHD, 17 (15.6\%) with autism, 15 (13.8\%) with developmental delays, 12 (11\%) with intellectual delays, and $12(11 \%)$ with language delays. Among the caregivers who participated in the survey interview, 82 were mothers (75.2\%), 19 (17.4\%) fathers, $6(5.5 \%)$ grandmothers and $2(1.8 \%)$ grandfathers.

All the caregivers were interviewed by a trained post-doctoral fellow in psychology. The study protocol was approved by the IRB of the university in the U.S. where the co-authors work. The consent form was explained by the interviewer and signed by the participants before the interview.

Table 1 summarizes relevant demographics for the families of the children in the study. There were approximately twice as many male as female children. With respect to the level of education, $91 \%$ of the mothers of children with delays had completed either university or junior college education. Most of the parents were married $(94.5 \%)$ with the divorce rate very low $(2.8 \%)$, and more than half of the families (66.1\%) were living with only their immediate family members, while $26.6 \%$ were living with other family members in addition to immediate family members.

Table 1: Descriptvie Statistics on the Characteristics of Sample Families $(n=109)$

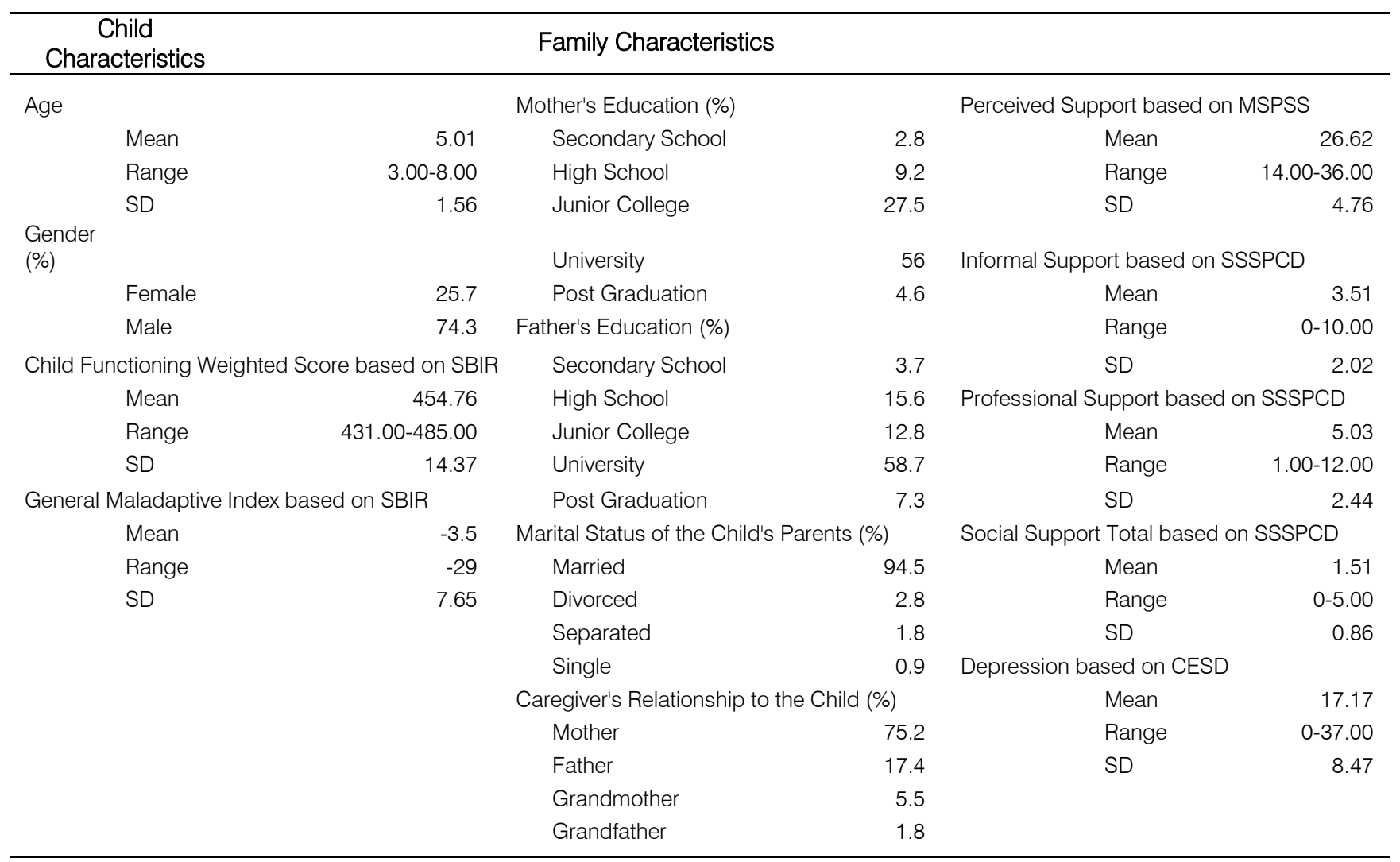


Family's Housing Status (\%)

Independent Housing

Shared Housing with Other

Family Members

Shared Housing with

Non-Family Members

Economic Status of the Family

Poor

Average

Rich
66.1

26.6

7.3

15.6

82.6

1.8
The economic status of the family was rated as being very poor, poor, average, or rich by the interviewer who conducted the home visit interviews and who examined the physical environment of the house. Families were considered rich when they had a large house with multiple floors and many high-quality possessions, such as cars, motorcycles, air conditioners, and a living room with a set of couches and a dining table set. When families had a spacious house with a robust structure and had enough possessions, such as motorcycles, refrigerators, televisions, and multi-rice cookers, they were considered middle income. Families were regarded as poor when their houses were not solid and not made of brick, had metal roofing, and some of the household materials were not of good quality. Families were considered very poor when their houses were made up of poor-quality brick and bamboo wattle with metal roofing or when they were living in the house of their relatives. Families that were considered very poor also did not have enough household materials. The majority of the families (82.6\%) were rated as middle income, followed by poor (15.6\%) and rich (1.8\%). None of the families were rated as very poor.

\section{ili. MeAsures}

Demographic characteristics: We included information on the child's age, gender and diagnosis. We asked about the education, occupation and economic status of the available caregivers.

Social support: We measured both the informal and professional support available to the caregivers as well as their perceived social support. The Social Support Scale for Parents of Children with Developmental Disabilities (SSSPCD) $)^{15}$ was used to assess the availability of informal and professional support for families of children with developmental delays in Vietnam. This instrument was adapted so that it would be culturally relevant to Vietnamese families ${ }^{11}$ and could be easily administered by professionals who work with these families. According to this instrument, 13 types of informal support and 8 types of professional support were available for Vietnamese families of children with developmental delays.
The sources of informal support consist of spouse, wife's father, wife's mother, husband's father, husband's mother, wife's siblings, husband's siblings, sons, daughters, other relatives, friends, neighbors, and other parents of children with developmental delays. Professional support can come from eight different sources: teachers, therapists, doctors, private therapists, home-helpers, social agencies, babysitters, and doctors in community health clinics. A source is designated as therapist or private therapist, doctor or doctor in a community health clinic depending on whether a fee is paid for the service. In this study, private therapists and doctors are those that require a fee.

The perceived social support was measured with the Multidimensional Scale of Perceived Social Support (MSPSS). ${ }^{16}$ The scale consists of 12 items that measure three components of social support: support from a special person, from family, or from a friend. The construct, convergent and discriminant validities of the scale have been demonstrated ${ }^{16}$, and it has adequate internal reliability (.88) and stability over time $(.95)^{17}$. The scale has been used in a variety of international contexts 18,19. We simplified the original seven Likert response categories to not at all (1), sometimes (2), and always (3). Possible scale values range from 12 to 36 , with a higher score indicating more perceived social support. The Cronbach alpha reliability of the instrument in this study was 85 .

Depression: Depression among caregivers was measured by using the Center for Epidemiologic Studies Depression Scale (CES-D) ${ }^{20}$. CES-D is a 20 -item selfreport scale that assesses the frequency of reported depressive symptoms of caregivers in the previous week (e.g., "I was bothered by things that don't usually bother me."). The CES-D is a widely used and internationally adapted scale for screening and assessing depressive symptoms in outcome studies of caregivers as well as of general and patient populations. The scale also has well-established reliability and discriminant validity for general and patient populations. The reliability of the scale in this sample was .74.

Child Behavior: The Scales of Independent BehaviorRevised Early Development Form (SIB-R) ${ }^{14}$ was used to 
assess the child's adaptive and maladaptive behavior. Parents answered 40 items regarding their child's adaptive behavior, including communication, eating, dressing, and social and motor skills. Parents also were asked to indicate whether their child engaged in eight domains of problem behavior and to rate both the frequency and severity of the behavior. Standard scores on adaptive behavior and General Maladaptive Index (GMI) scores were calculated using the procedures outlined in the SIB-R manual. The reliability of the adaptive behavior scale in this sample was .95 and that of the maladaptive behavior scale .86 .

All of the scales used in this study except SIB-R were validated in Vietnamese ${ }^{11,12}$. In creating the Vietnamese version of SIB-R, the scale was evaluated for content and semantic equivalence of the Vietnamese and English versions. Content equivalence is established when the content of each item of the scale is relevant to the phenomena of each culture being studied ${ }^{21}$. Three bilingual Vietnamese rated each item on a 3-point scale as 1 (relevant to the Vietnamese culture), 2 (somewhat relevant to the Vietnamese culture) or 3 (not relevant to the Vietnamese culture). The few items that were rated as somewhat relevant were considered for rewording. Reworded items were re-examined using the same technique. For evaluation of semantic equivalence of the measurement, three bilingual Vietnamese rated each item on a 3-point scale, from 1 (exactly the same meaning in both versions), 2 (almost the same meaning in both versions) to 3 (different meaning in each version). Items rated as different meaning were considered for rewording, and reworded items were re-examined using the same technique.

\section{Results}

\section{a) Social support}

The caregivers scored an average of 3.5 ( $S D=$ 2.02) out of a possible 13 on the SSSCDD subscale that measures the amount of available informal support and $1.5(S D=.86)$ out of a possible 8 on the subscale that measures the amount of available professional support. The largest proportion of informal support was from spouse (91.7\%), followed by husband's mother (52.3\%), wife's mother (41.3\%), husband's father (37.6\%), wife's father $(29.4 \%)$, wife's sibling $(17.4 \%)$, parents of other children with delays $(16.5 \%)$, friend $(16.5 \%)$, husband's sibling (15.6\%), neighbor(13.8\%), son (7.3\%), daughter (5.5\%), and other relative (3.7\%). The largest proportion of professional support was from private therapist $(91.7 \%)$, followed by teacher (27.5\%), doctor, (12.8\%), home help (7.3\%), hospital therapist (6.4\%), doctor in community clinic (2.8\%), and babysitter (1.8\%). For informal support, the majority of the caregivers were relying on support from their spouse, and a significant proportion relied on their own parents and parents-inlaw to help raise the children with delays. For professional support, almost all the families hired a private therapist to work with their children. In contrast, only a quarter of the families stated that teachers were available to educate their children. Those with a greater amount of informal support tended to have a greater amount of professional support available to them, $r$ $(105)=.32, p<.001$.

Table 2: Types and availability of informal and formal social support $(n=109)$

\begin{tabular}{lcc}
\hline & Availability & Percent (\%) \\
\hline Informal Support & & \\
Spouse & 100 & 91.70 \\
Wife's Father & 32 & 29.40 \\
Wife's Mother & 45 & 41.30 \\
Husband's Father & 41 & 37.60 \\
Husband's Mother & 57 & 52.30 \\
Husband's Sibling(s) & 17 & 15.60 \\
Wife's Sibling(s) & 19 & 17.40 \\
Son(s) & 8 & 7.30 \\
Daughter(s) & 6 & 5.50 \\
Other Relative(s) & 4 & 3.70 \\
Friend(s) & 18 & 16.50 \\
Neighbor(s) & 15 & 13.80 \\
Parents of Other Children with Delays & 18 & 16.50 \\
Formal Support & & \\
Teacher & 30 & 27.50 \\
Hospital Therapist & 7 & 6.40 \\
Doctor & 14 & 12.80 \\
Private Therapist & 100 & 91.70 \\
Home Help & 8 & 7.30 \\
Babysitter & 2 & 1.80 \\
Social Agency & 0 & 0 \\
Doctor in Community Clinic & 3 & 2.80 \\
\hline
\end{tabular}


There was a significant relationship between the amount of informal support and the amount of perceived support based on the MSPSS, $r(106)=.19, p<.05$. The caregivers who listed more people as being available to provide informal support perceived a higher level of social support. However, the amount of professional support was not related to perceived social support, $r(106)=.12, p>.05$.

\section{b) Depression}

The degree of depression reported by the caregivers is alarming, with the mean CES-D score elevated beyond the cut-off point of 16 that indicates a risk of clinical depression $(M=17.17 S D=8.47)$. Out of 109 caregivers, 61 (56\%) were suspected of being clinically depressed. We further investigated the characteristics of these caregivers by examining the diagnoses of their children. Unfortunately, 42 (19\%) out of the 109 caregivers did not know their child's diagnosis. Among those who did, the caregivers' depression was compared across different diagnosis groups. The caregivers of children with ADHD were most depressed $(M=19.82, S D=8.60)$, followed by caregivers of children with autism $(M=17.41, S D=$ 9.34), developmental delays $(M=15.40, S D=6.99)$, intellectual delays $(M=15.83, S D=8.41)$ and language delays $(M=13.08, S D=10.28)$, although there is no significant difference among the four groups, $F(4,62)=.96, p>.05$. Among the caregivers whose children were diagnosed with ADHD, 8 out of 11 scored beyond the cut-off point of 16 (72.7\%), while 12 out of 17 caregivers whose children were diagnosed with autism scored above 16 (58.7\%). Among those caregivers whose children were diagnosed with developmental delays, 7 out of 15 scored above 16 (46.7\%). Among those whose children were diagnosed with intellectual delays, 4 out of 12 (33.2\%) scored above 16, and of those whose children were diagnosed with language delays, 4 out of 12 (33.2\%) scored above 16. For a significant proportion of the caregivers of children with ADHD and autism, the long-term demands of caregiving appear to have severely affected their mood.

\section{c) Social Support and Depression}

It was hypothesized that the caregivers who received higher levels of social support would be less depressed. The intercorrelations between the variables were computed by conducting Pearson Product Moment Correlations and are presented in Table 3. There was no significant correlation between the total scores for informal support based on SSSCDD and the total scores for depression based on CESD, $r$ (106) = $.13, p>.05$. There was no significant correlation between the total scores for professional support based on SSSCDD and the total scores for depression based on CESD, $r(106)=-.12, p>.05$. The amount of informal and professional support, which was measured by assessing the number of people who were available to provide support, was not significantly related to the caregivers' experience of depression. There was a significant correlation between the total scores for perceived support based on MSPSS and the total scores for depression based CESD $(r(107)=-.31$, $p<.001$ ), suggesting that the caregivers who perceived a lower level of social support experienced a higher level of depression.

\section{d) Correlations among psychosocial, child and demographic variables}

The intercorrelations between the demographic, child and psychosocial variables were computed to examine the relationships between psychosocial variables and child and demographic variables (Table 3). Caregivers' depression was significantly related to the child's maladaptive behavior, family socioeconomic status and mother's education. Caregivers who had children with more maladaptive behaviors experienced a higher level of depression than those whose children exhibited fewer maladaptive behaviors, $r(106)=-.31$, $p<.001$. The caregivers experienced elevated depression when their families had a lower socioeconomic status $(r(107)=-.20, p<.05)$ and when the children's mothers were less educated, $r(107)=-$ $.19, p<.05$.

Caregivers were more likely to perceive a higher level of social support when their children were younger $(r(107)=-.23, p<.05)$, the mother had obtained a higher level of education $(r(107)=.20, p<.05)$, and the family had a higher socioeconomic status, $r(107)=.19$, $p<.05$. However, the perceived social support was not related to the adaptive or maladaptive status of the children. Neither the amount of informal support nor that of professional support was related to child's adaptive and maladaptive functioning, mother's education, or socioeconomic status of the family.

\section{e) Predictors of depression among caregivers}

The four variables that correlated significantly with depression (child's maladaptive behavior, mother's education, socioeconomic status, and perceived social support) were entered into a hierarchical regression analysis to determine the best set of predictors of depression among caregivers (Table 4). The first step included the child's maladaptive behavior as a stressor. The other variables (mother's education, socioeconomic status and perceived social support) were entered into the regression as family resources to determine if they entered significantly in a second step of the hierarchical regression. Table 3 shows the four variables that were entered into two steps. The first predictor variable accounted for nearly $10 \%$ of the variance in depression scores $(F(1,107)=11.48, p<.001)$. Child's maladaptive behavior accounted for a significant amount of the variance in depression among caregivers. Mother's education, socioeconomic status, and 
perceived social support also entered the model significantly, accounting for an additional $8 \%$ of the variance in stress scores $(F(4,104)=5.53, p<.001)$. When all four independent variables were included in the second step, neither socioeconomic status nor maternal education was a significant predictor of depression. The most important and significant predictor of caregivers' depression was perceived social support followed by child's maladaptive behavior.

\section{Discussion}

The present study explored the linkage between depression and social support among the caregivers of young children with developmental delays in Vietnam. The level of depression experienced by Vietnamese caregivers of children with developmental delays was affected by their perceived social support. The more the caregivers felt that they received support, especially from family, friends or somebody special, the less depression they experienced. This finding is consistent with those of previous research on the relationship between social support and depression ${ }^{22,23}$. However, when social support was measured in terms of the amount of support available to the caregivers, neither informal nor professional support had a significant impact on depression, implying that the mere number of people available does not significantly affect the caregivers' mood. Only when the caregivers really felt that they were cared for did the amount of social support show a significant relationship to mood.

The extent of maladaptive behavior had a significant impact on depression among the caregivers. The impact of maladaptive behavior of the children on the stress levels and mood of the caregivers has been consistently documented in many of the previous studies $5,22-24$. Beyond and above the intellectual and adaptive challenges their children bring to the caregiving, when the children show impulsive, aggressive or unruly behaviors, this has a significant influence on the mood of the caregivers, suggesting that the demands of coping with the children's maladaptive behaviors have a long-term impact on the quality of the everyday mood they experience.

In addition, the level of the caregivers' depression in Vietnam is alarming, with more than half of them scoring at a level consistent with clinical depression. Although the ways in which depressive symptoms are expressed can vary from culture to culture, many Vietnamese caregivers appear to suffer far more severely than those in Western culture: The prevalence rate of depression has been reported at 20\% to $30 \%$ among US mothers of children with intellectual disabilities ${ }^{5,22,25}$. In particular, a significant proportion of Vietnamese caregivers of children with ADHD (72.7\%) and autism $(58.71 \%)$ suffer from this suspected clinical level of depression, revealing that children with behavioral problems impose long-term demanding caregiving burdens to such an extent that their caregivers' mood deteriorates. Depression, especially at clinical levels, is about losing a sense of meaning and motivation in everyday life. The impact on the caregivers was such that coping with their children was not just overwhelming and difficult, but they felt that there was no hope and fun in their lives, only the interminable sadness, helplessness, hopelessness and exhaustion of coping with their difficult children and not getting

Table 3: Correlations among variables

\begin{tabular}{|c|c|c|c|c|c|c|c|c|c|c|c|c|}
\hline Variables & 1 & 2 & 3 & 4 & 5 & 6 & 7 & 8 & 9 & 10 & 11 & 12 \\
\hline \multicolumn{13}{|l|}{ 1. Age } \\
\hline 2. Child's Gender & 0.08 & & & & & & & & & & & \\
\hline 3. Mother's Education & -0.18 & 0.05 & & & & & & & & & & \\
\hline 4. Father's Education & -0.06 & 0.05 & $0.71^{\star \star}$ & & & & & & & & & \\
\hline $\begin{array}{l}\text { 5. Economic Status of the } \\
\text { family }\end{array}$ & -0.12 & 0.21 & $0.32^{\star \star}$ & 0.00 & & & & & & & & \\
\hline 6. Perceived Social Support & -0.23 & 0.06 & $0.20 *$ & 0.28 & $0.19 *$ & & & & & & & \\
\hline 7. Parent Depression & 0.07 & 0.01 & $-0.19^{\star}$ & 0.16 & $-0.20^{*}$ & $-0.31 * *$ & & & & & & \\
\hline 8. Social Support Informal & -0.02 & -0.05 & -0.01 & 0.87 & 0.00 & $0.19^{\star}$ & -0.13 & & & & & \\
\hline $\begin{array}{l}\text { 9. Social Support } \\
\text { Professional Total }\end{array}$ & 0.00 & -0.08 & -0.10 & 0.53 & 0.15 & 0.12 & -0.12 & $0.32^{\star *}$ & & & & \\
\hline 10. Social Support Total & -0.01 & -0.07 & -0.04 & 0.73 & 0.05 & $0.20^{*}$ & -0.14 & $0.94^{* *}$ & $0.62^{\star *}$ & & & \\
\hline 11. Parent Mental Health & 0.00 & 0.07 & $-0.20^{\star}$ & 0.07 & $-0.26^{* *}$ & -0.11 & $0.53^{\star *}$ & 0.05 & 0.02 & 0.05 & & \\
\hline $\begin{array}{l}\text { 12. Child Functioning } \\
\text { Weighted Score }\end{array}$ & $0.40^{* *}$ & -0.10 & 0.13 & 0.53 & $-0.20^{*}$ & 0.08 & -0.15 & 0.14 & 0.18 & 0.19 & 0.01 & \\
\hline $\begin{array}{l}\text { 13. General Maladaptive } \\
\text { Index }\end{array}$ & -0.22 & -0.03 & $0.25^{\star \star}$ & 0.46 & $0.28^{* *}$ & 0.12 & $-0.31^{\star *}$ & -0.13 & -0.08 & -0.14 & $-0.30^{* *}$ & -0.14 \\
\hline
\end{tabular}

Note. ${ }^{\star} p<.05 ;{ }^{* \star} p<.01 ;$ two-tailed tests 
Table 4: Regression model: Predictor Variables of Depression $(n=109)$

\begin{tabular}{|c|c|c|c|c|c|c|}
\hline Independent variable & $B$ & $S E$ & Standardized B & & $F$ & $R^{R}$ Change \\
\hline Step 1 & & & & & 11.48 & $0.10^{* * *}$ \\
\hline Child's maladaptive behavior & -0.35 & 0.10 & -0.31 & $* \star *$ & & \\
\hline Step 2 & & & & & 5.53 & $0.08^{*}$ \\
\hline Child's maladaptive behavior & -0.26 & 0.11 & -0.24 & ** & & \\
\hline Family SES & -1.39 & 2.08 & -0.07 & & & \\
\hline Mother's education & -0.60 & 0.98 & 0.06 & & & \\
\hline Perceived social support & -0.44 & 0.16 & -0.25 & ** & & \\
\hline
\end{tabular}

Note. ${ }^{*} p<.05 ;{ }^{* *} p<.01 ;{ }^{* *} p<.001 ;$ two-tailed tests.

the support they needed. These were caregivers of young children: When the caregivers' daily mood is affected this severely when their children are young, we can only imagine how they will fare as their children advance in age without having their behavior issues addressed. It is paramount that the caregivers of children with behavioral issues obtain support to manage the daily issues arising from their children's maladaptive behaviors.

When we examined the availability of informal support, we found that caregivers mostly relied on their spouses for help as well as on both the mothers' and fathers' parents. Although the availability of informal support was not related to depression, it was related to perceived support. Having a large enough number of people around to provide support to the caregivers could be helpful in their perception of social support, which in turn might help with the depressive symptoms. Regarding professional support, almost all caregivers (91.7\%) reported the availability of private therapists. These therapists could be professional therapists, but in an attempt to advance their children to an upper level in school (the kindergarten programs in Vietnam hold the children to repeat the grade until they are qualified to advance to elementary schools), they tended to hire many private tutors, who were often college or high school students without training in special needs, to help their children to master academic materials. This could be a costly and inadequate investment: The children with cognitive and attention issues might resent having to work on demanding academic materials just to move on to the advanced level. A quarter of the caregivers (27.5\%) reported that teachers were available, which is disappointing considering that all these children were enrolled in kindergarten programs. It is clear that many teachers do not have enough skills to work with these children. There should be more formal training programs available to train the teachers to work with the children with special needs in integrated and inclusive settings of kindergarten programs in Vietnam. While many caregivers are severely affected by their children's condition, especially when they have behavioral issues, there should be more professional support systems that could train and provide teachers who could address these issues.

Mother's education was significantly related to depression and perceived social support. When the mothers were more educated, the caregivers experienced less depression and perceived a higher level of social support. When mothers are highly educated, they tend to attain higher socioeconomic status, enabling them to utilize the greater financial resources to better care for their children. In addition, they have more intellectual resources, which can enable them to develop better strategies to cope with the children's demanding cognitive and behavioral issues and thus to experience less depression. They may be able to make more effective use of the available social support in raising their children. While general support services should be available to all the families, there should be more support and education available for the mothers with less education and financial resources. Helping mothers to build and strengthen coping strategies and informal support systems could be critical for the well-being of the caregivers, and this needs to be addressed when the programs for young children provide the services. The findings also suggest that there is a need for services and supports that help caregivers not only to be effective parents, but also that address feelings of distress that stem from parenting children with disabilities. This support needs to come from professionals who can provide mental health counseling or psychotherapy, helping the caregivers develop effective personal coping strategies.

The study has a few limitations. Our project was geographically limited to Hanoi, the capital city and one of the biggest cities in Vietnam. Most of our participants were relatively well educated and the majority had completed their college degrees. In addition, although developing, the resources for children with special needs are relatively concentrated in big cities like Hanoi, where the families could rely on professional help to take care of their children more than could those residing in smaller cities or countries. We suspect that 
parents with less education and with fewer financial resources in other areas of Vietnam are in a direr situation in coping with the issues their children impose on them.

As in many other Asian cultures, negative perceptions and attitudes prevail in Vietnam toward individuals with disabilities. These perceptions and attitudes are often extended to their caregivers and families, who may be perceived as not raising their children properly ${ }^{10,11}$. While there should be environmental support for changing the attitudes toward those with disabilities, the experience of stigma should be explored in future studies as impacting the caregivers' mood and diminishing the quality of life that individuals with disabilities and their families deserve.

\section{ACKNOWLEDGEMENTS}

This project was supported by an HCLAS Faculty Research and Development Grant from Hofstra University, USA.

\section{References Références Referencias}

1. Cantwell J, Muldoon O, Gallagher S. The influence of self-esteem and social support on the relationship between stigma and depressive symptomology in parents caring for children with intellectual disabilities. Journal of Intellectual Disability Research. 2015; 59(10): 948-957. doi:10.1111/jir.12205

2. Estes A, Munson J, Dawson G, Koehler E, Zhou X$\mathrm{H}$, Abbott R. Parenting stress and psychological functioning among mothers of preschool children with autism and developmental delay. Autism. 2009; 13(4): 375-387. doi:10.1177/1362361309105658

3. Olsson MB, Hwang CP. Depression in mothers and fathers of children with intellectual disability. Journal of Intellectual Disability Research. 2001; 45(6): 535543. doi:10.1046/j.1365-2788.2001.00372.x

4. Olsson MB, Hwang CP. Socioeconomic and psychological variables as risk and protective factors for parental well-being in families of children with intellectual disabilities. Journal of Intellectual Disability Research. 2008; 52(12): 1102-1113. doi:10.1111/j.1365-2788.2008.01081.x

5. Zeedyk SM, Blacher J. Longitudinal Correlates of Maternal Depression Among Mothers of Children With or Without Intellectual Disability. American Journal on Intellectual and Developmental Disabilities. 2017; 122(5): 374-391. doi: 10.1352/ 1944-7558-122.5.374.

6. Falk NH, Norris K, Quinn MG. The factors predicting stress, anxiety and depression in the parents of children with autism. Journal of autism and developmental disorders. 2014; 44(12): 3185-3203.
7. Halstead EJ, Griffith GM, Hastings RP. Social support, coping, and positive perceptions as potential protective factors for the well-being of mothers of children with intellectual and developmental disabilities. International Journal of Developmental Disabilities. 2018; 64(4-5): 288-296. doi:10.1080/20473869.2017.1329192.

8. Zaidman-Zait A, Mirenda P, Duku E, et al. Impact of personal and social resources on parenting stress in mothers of children with autism spectrum disorder. Autism. 2017; 21(2):155-166.

9. Oh H, Lee EO. Caregiver burden and social support among mothers raising children with developmental disabilities in South Korea. International Journal of Disability, Development and Education. 2009; 56(2): 149-167.

10. Hunt PC. An introduction to Vietnamese culture for rehabilitation service providers in the United States. Culture and disability: Providing culturally competent services. Published online 2005:203-223.

11. Shin J, Nhan NV, Crittenden KS, Hong, HTD, Flory, $M$, \& Ladinsky J. Parenting stress of mothers and fathers of young children with cognitive delays in Vietnam. Journal of Intellectual Disability Research. 2006; 50(10):748-760.

12. Shin JY, Nhan NV. Predictors of parenting stress among Vietnamese mothers of young children with and without cognitive delay. Journal of Intellectual and Developmental Disability. 2009; 34(1):17-26.

13. Thuy NTM, Berry HL. Social capital and mental health among mothers in Vietnam who have children with disabilities. null. 2013; 6(1): 18886. doi:10.3402/gha.v6i0.18886

14. Bruininks RH, Woodcock RW, Weatherman RF, Hill BK. Scales of Independent Behavior-Revised SIB-R. Riverside Publishing; 1996.

15. Shin JY, Crittenden KS. Social Support Scale for Parents of Children with Disabilities. Published online 1997.

16. Zimet GD, Dahlem NW, Zimet SG, Farley GK. The Multidimensional Scale of Perceived Social Support. null. 1988; 52(1): 30-41. doi:10.1207/s15327752 jpa52012

17. Cecil $\bar{H}$, Stanley MA, Carrion PG, Swann A. Psychometric properties of the MSPSS and NOS in psychiatric outpatients. Journal of Clinical Psychology. 1995; 51(5): 593-602. doi:10.1002/ 1097-4679(199509)51:5<593::AID-JCLP22705 10503>3.0.CO;2-W

18. Chou K-L. Assessing Chinese adolescents' social support: the multidimensional scale of perceived social support. Personality and Individual Differences. 2000; 28(2): 299-307. doi:10.1016/ S0191-8869(99)00098-7

19. Duru E. Re-examination of the psychometric characteristics of the multidimensional scale of perceived social support among Turkish university 
students. Social Behavior and Personality: an international journal. 2007; 35(4):443-452.

20. Radloff LS. The CES-D Scale: A Self-Report Depression Scale for Research in the General Population. Applied Psychological Measurement. 1977; 1(3): 385-401. doi:10.1177/014662167700 100306

21. Liang B, Bogat GA. Culture, control, and coping: New perspectives on social support. American Journal of Community Psychology. 1994; 22(1):123147. doi:10.1007/BF02506820

22. Bailey DBJr, Golden RN, Roberts J, Ford A. Maternal depression and developmental disability: Research critique. Mental Retardation and Developmental Disabilities Research Reviews. 2007; 13(4): 321-329. doi:10.1002/mrdd.20172

23. Feldman M, McDonald L, Serbin L, Stack D, Secco ML, Yu CT. Predictors of depressive symptoms in primary caregivers of young children with or at risk for developmental delay. Journal of Intellectual Disability Research. 2007; 51(8): 606-619. doi:10.1111/j.1365-2788.2006.00941.x

24. Gallagher S, Phillips AC, Lee H, Carroll D. The association between spirituality and depression in parents caring for children with developmental disabilities: Social support and/or last resort. Journal of Religion and Health. 2015; 54(1): 358-370. doi:10.1007/s10943-014-9839-x

25. Singer GHS. Meta-Analysis of Comparative Studies of Depression in Mothers of Children with and Without Developmental Disabilities. Floyd F, ed. American Journal on Mental Retardation. 2006; 111(3): 155-169. doi: 10.1352/0895-8017(2006) 111[155:MOCSOD]2.0.CO;2 